ARTICLE

DOI: $10.1038 /$ s41467-018-07644-6

\title{
GSK3 suppression upregulates $\beta$-catenin and c-Myc to abrogate KRas-dependent tumors
}

\author{
Aslamuzzaman Kazi ${ }^{1}$, Shengyan Xiang ${ }^{1}$, Hua Yang ${ }^{1}$, Daniel Delitto ${ }^{2}$, José Trevino ${ }^{2}$, Rays H.Y. Jiang ${ }^{3}$, \\ Muhammad Ayaz ${ }^{1}$, Harshani R. Lawrence ${ }^{1}$, Perry Kennedy ${ }^{1} \&$ Saïd M. Sebti (i) ${ }^{1,4}$
}

Mutant KRas is a significant driver of human oncogenesis and confers resistance to therapy, underscoring the need to develop approaches that disable mutant KRas-driven tumors. Because targeting KRas directly has proven difficult, identifying vulnerabilities specific for mutant KRas tumors is an important alternative approach. Here we show that glycogen synthase kinase 3 (GSK3) is required for the in vitro and in vivo growth and survival of human mutant KRas-dependent tumors but is dispensable for mutant KRas-independent tumors. Further, inhibiting phosphorylation of GSK3 substrates c-Myc on T58 and $\beta$-catenin on S33/ S37/T41 and their subsequent upregulation contribute to the antitumor activity of GSK3 inhibition. Importantly, GSK3 blockade inhibits the in vivo growth of G12D, G12V, and G12C mutant KRas primary and metastatic patient-derived xenografts from pancreatic cancer patients who progressed on chemo- and radiation therapies. This discovery opens new avenues to target mutant KRas-dependent cancers.

\footnotetext{
${ }^{1}$ Drug Discovery Department, H. Lee Moffitt Cancer Center and Research Institute, Tampa, FL 33612, USA. ${ }^{2}$ Department of Surgery, University of Florida, Gainesville, FL 32610, USA. ${ }^{3}$ Department of Global Health and Center for Drug Discovery and Innovation, University of South Florida, Tampa, FL 33612, USA. ${ }^{4}$ Chemical Biology and Molecular Medicine Program, H. Lee Moffitt Cancer Center and Research Institute, Tampa, FL 33612, USA. These authors contributed equally: Aslamuzzaman Kazi, Shengyan Xiang. Correspondence and requests for materials should be addressed to S.M.S. (email: Said.Sebti@moffitt.org)
} 
as proteins are low-molecular-weight GTP/GDP-binding GTPases that, under physiological conditions, regulate several important cellular processes, including cell growth, differentiation, and survival ${ }^{1,2}$. There are three human Ras genes that encode HRas, NRas, and KRas proteins. Ras proteins mediate the transfer of biological information from cell surface receptors to intracellular signaling pathways such as the Raf/Mek/Erk, PI3K/Akt, RalGDS/Ral, TIAM1/Rac, and p190/Rho pathways, eventually leading to regulation of gene expression, cell cycle progression, survival, cytoskeletal changes, and motility ${ }^{2}$.

Since their identification in mammalian cells in 1981, Ras genes have been shown to play pivotal roles in human tumor pathogenesis, contributing to several hallmarks of human cancer and driving tumorigenesis in genetically engineered mouse models ${ }^{1,2}$. Clinically, KRas, the most frequently mutated Ras gene, confers resistance to therapy in cancers such as pancreatic, colon, and lung ${ }^{1,2}$. Notably, patients with mutant KRas cancers have poor prognosis, increased tumor aggressiveness and metastasis, and are less likely to respond to chemotherapy and targeted therapies ${ }^{3-6}$, leading the National Comprehensive Cancer Network to recommend treatment with epidermal growth factor (EGF) receptor inhibitors only in patients whose tumors harbor wild-type KRas ${ }^{4}$. These observations prompted many to target mutant KRas, which unfortunately has proven to be difficult. Although recent efforts to understand the conformational changes and dynamics of KRas resulted in the identification of covalent as well as non-covalent binders of $\mathrm{KRas}^{7-9}$, currently there are no approved therapies that directly target mutant $\mathrm{KRas}^{10}$. However, mutant KRas-driven cancers may gain dependencies through other pathways ${ }^{11}$. Here, by exploring vulnerabilities of human tumors that depend on mutant KRas, we sought to identify kinases and their corresponding pathways that mutant KRas depends on to induce malignant transformation and to target such pathways for cancer therapy.

\section{Results}

GSK3 is required for survival of KRas-dependent tumors. To identify kinase inhibitors that selectively suppress the viability of human cancer cells that depend on mutant KRas, we first screened a 304-compound, well-cured kinome inhibitor library, the GlaxoSmithKline Published Kinase Inhibitor Set $1^{12}$, against human pancreatic (MiaPaCa2) and lung (A549) cancer cells. Although both MiaPaCa2 and A549 cell lines harbor mutant KRas, previous work showed these cell lines to be mutant KRasdependent and -independent, respectively ${ }^{13,14}$, and we confirmed their dependency status in cell culture (Fig. 2) and in mice (Fig. 3). After cells were treated with the 304 kinase inhibitors (1 $\mu \mathrm{M})$ for $72 \mathrm{~h}$ in 96-well plates using a "one well-one kinase inhibitor" format, we determined the difference in percent inhibition of viability $\left[D=\left(\begin{array}{lll}\% & \mathrm{MiaPaCa} 2 & \text { inhibition }\end{array}\right)-\left(\begin{array}{ll}\% & \mathrm{~A} 549\end{array}\right.\right.$ inhibition)] for each compound based on the average of two screens. Figure la shows that $197 / 304$ compounds (65\%) affected $\mathrm{MiaPaCa} 2$ and $\mathrm{A} 549$ cell viability equally, with $D$ values from $-10 \%$ to $+10 \%$. The GSK3 $\alpha / \beta$ inhibitor SB-732881-H (SB) (Fig. 1a, inset) had the highest selectivity for inhibiting the viability of MiaPaCa2 versus A549 cells $(D=81 \%)$, inhibiting MiaPaCa2 cells by 85 and $80 \%$ (two independent screens) but A549 cells by only 1 and 3\% (Fig. 1b). We synthesized SB inhouse to confirm these findings, showing that $\mathrm{SB}$ inhibited $\mathrm{MiaPaCa} 2$ versus $\mathrm{A} 549$ cell viability with a 45 -fold lower $\mathrm{IC}_{50}(0.4$ versus $18 \mu \mathrm{M}$; Fig. 1c). Other GSK3 inhibitors (Tideglusib, AZD1080, and BIO) with chemical structures different from SB showed similar selectivity (Supplementary Fig. 1, top), confirming that MiaPaCa2 cells are more sensitive than A549 cells to inhibition of GSK3 $\alpha / \beta$. Furthermore, SB induced caspase- 3 activation and PARP cleavage only in MiaPaCa2 but not in A549 cells (Fig. 1d), suggesting that GSK3 $\alpha / \beta$ inhibition selectively induces apoptosis in mutant KRas-dependent tumor cells. Kinome profiling at $100 \mathrm{nM}$ showed that SB is highly selective for GSK3 $\alpha / \beta$, with over $96 \%(216 / 224)$ of the kinases inhibited by $<10 \%$ (data from ref. ${ }^{9}$ plotted as Supplementary Fig. 1, bottom; with permission from Nature Biotechnology). GSK $3 \alpha$ and GSK3 $\beta$ were the two most potently inhibited kinases ( $80 \%$ and $77 \%$, respectively). Furthermore, unlike GSK3 inhibition with SB that suppresses the viability of the mutant KRas-dependent MiaPaCa2 cells selectively over that of the mutant KRas-independent A549 cells, inhibition of other kinases does not. For example, treating Mia$\mathrm{PaCa} 2$ and A549 cells with the PLK1 kinase inhibitor, GSK317314A $\mathrm{A}^{12}$, or the dual PLK1 and LOK inhibitor, GSK237701 $\mathrm{A}^{12}$, inhibited equally the viability of both cell lines (Supplementary Fig. 2). The MAPK3 inhibitor, GW301789X ${ }^{12}$, and the ErbB4 kinase inhibitor, GR269666A ${ }^{12}$, had little effects on the viability of neither MiaPaCa2 nor A549 cells (Supplementary Fig. 2). Furthermore, the multi-kinase inhibitor GW780056X (ARK5, KIT, CDK4, HIPK1, CLK2, DYRK1, and CDK2) ${ }^{12}$ inhibited equally the viability of MiaPaCa2 and A549 cells, whereas another multi-kinase inhibitor, GSK619487A (PKC, AKT1, IKK, PKA, AKT2, and AKT3) ${ }^{12}$, had little effect on the viability of either cell line (Supplementary Fig. 2).

These findings support the hypothesis that mutant KRasdependent human cancer cells are vulnerable to GSK $3 \alpha / \beta$ inhibition and that mutant KRas-dependent cells may require GSK $3 \alpha / \beta$ for tumor survival. To further investigate this hypothesis, we used SMARTpool siGENOME small interfering RNAs (siRNAs) to deplete KRas and GSK $3 \alpha / \beta$ from a panel of eight mutant KRas-harboring human cancer cell lines and then determined the effects of silencing these genes on apoptosis and viability. KRas depletion induced caspase-3 activation and PARP cleavage in MiaPaCa2 and L3.6pl (pancreatic), SW620 (colon), and Calu-6 (lung) cells but did not in A549 and H460 (lung) and DLD-1 and HCT-8 (colon) human cancer cells (Fig. 2a), indicating that the former four tumor cell lines are mutant KRas-dependent, whereas the latter four cell lines are not. Importantly, GSK $3 \alpha / \beta$ depletion only induced apoptosis in cell lines that were mutant KRas-dependent (Fig. 2a). Similarly, GSK3 $\alpha / \beta$ depletion only inhibited viability in cell lines where depletion of KRas inhibited viability (Supplementary Fig. 3a). Consistent with these findings, pharmacological inhibition of GSK $3 \alpha / \beta$ with SB only induced apoptosis and inhibited viability in cell lines where depletion of KRas induced apoptosis and inhibited viability (Fig. 2b and Supplementary Fig. 3b). Notably, SB did not affect the viability of human non-malignant immortalized cells from kidney (HEK293), pancreas (human pancreatic epithelial nestin-expressing; HPNE), and ovarian (T80) origin (Supplementary Fig. 3b). In addition, SB was not able to induce apoptosis in eight human cancer cell lines that harbor wild-type KRas (H661, H2126, H322, H1299, H522, PC9, H4006, and DU145) as compared to those that harbor mutant KRas (Supplementary Fig. 3c). Finally, Supplementary Fig. 3d shows that SB treatment induced apoptosis in control empty vector cells as well as cells ectopically expressing wildtype GSK3. In contrast, ectopic expression of the constitutive active GSK3-S9A mutant compromised the ability of SB to induce apoptosis. Together, the above findings support the hypothesis that mutant KRas-dependent human cancer cells are vulnerable to GSK $3 \alpha / \beta$ inhibition and that, in these cells, mutant KRas requires GSK3 $\alpha / \beta$ to maintain tumor cell survival.

GSK3 or KRas silencing inhibits in vivo growth of KRasdependent tumors. To evaluate the effects of SB on tumor 

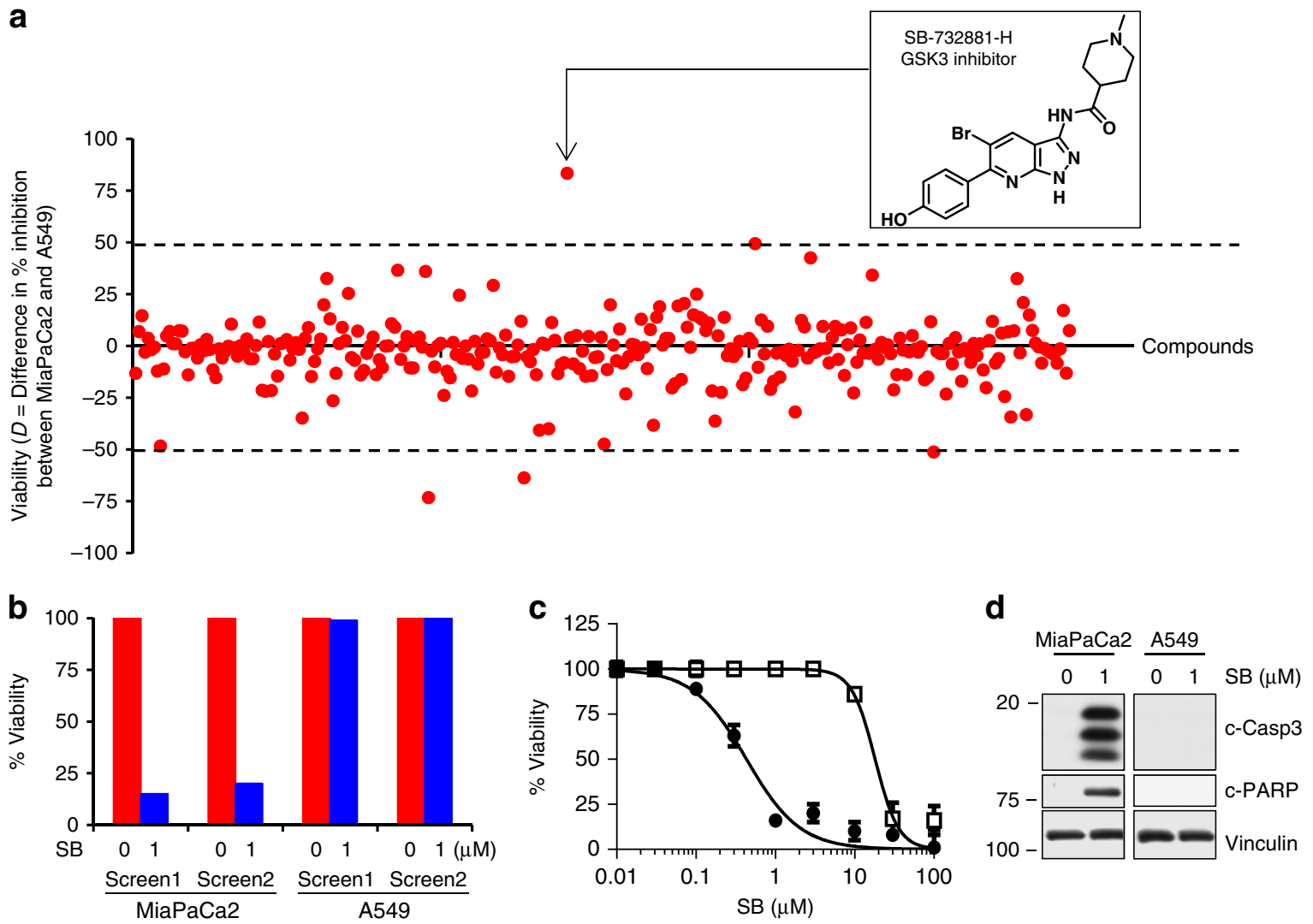

Fig. 1 Kinome screen identifies GSK3 inhibitor that suppresses mutant KRas-dependent cells. a MiaPaCa2 (mutant KRas-dependent) and A549 (mutant KRas-independent) human tumor cells were treated for $72 \mathrm{~h}$ in 96 -well plates with 304 kinase inhibitors ( $1 \mu \mathrm{M}$ ) using a one well-one inhibitor format. $D[(\%$ inhibition of viability of MiaPaCa2) - (\% inhibition of viability of A549)] was determined for each compound based on the average of two screens. b Effects of SB on percent cell viability from both screens. $\mathbf{c} I C_{50}$ determination of in-house synthesized SB (experiment done three times). $\mathbf{d}$ Western blots showing SB-induced caspase-3 activation and PARP cleavage in MiaPaCa2 but not A549 cells (experiment done three times)

growth in vivo, we subcutaneously implanted MiaPaCa2 and A549 cells on right and left flanks, respectively, of nude mice (Fig. 3a). After tumors reached $150-200 \mathrm{~mm}^{3}$, mice were treated daily with vehicle or SB ( $50 \mathrm{mg} / \mathrm{kg}$ intraperitoneally). SB treatment suppressed the growth of MiaPaCa2 but not A549 tumors (Fig. 3a-c). At day 21, MiaPaCa2 tumor growth was inhibited by $81 \%$ in SB-treated mice compared with vehicle-treated mice (Fig. 3b), whereas growth of A549 tumors was not inhibited (Fig. 3c). The difference in the average tumor volume between vehicle- and SB-treated MiaPaCa2 tumors was statistically significant starting at day 7 and remained so thereafter (Fig. 3b).

To determine whether depletion of either GSK $3 \alpha / \beta$ or KRas affects MiaPaCa2 and A549 tumor growth in mice, we transfected MiaPaCa2 and A549 cells with non-targeting (NT), KRas, or GSK $3 \alpha / \beta$ siRNA for $48 \mathrm{~h}$ and then subcutaneously implanted equal numbers of harvested cells on right (MiaPaCa2) and left (A549) flanks. An aliquot from the same cells used for implantation was analyzed by western blot to confirm depletion (Fig. 3d, e, insets). Compared with control NT siRNA, knockdown of KRas in MiaPaCa2, but not in A549, cells inhibited tumor growth in mice, confirming in vivo the dependency of MiaPaCa2 but not A549 tumors on KRas. Importantly, depletion of GSK3 $\alpha / \beta$ inhibited MiaPaCa2 but not A549 tumor growth, further supporting the hypothesis that mutant KRas-dependent but not -independent tumors require GSK3 $\alpha / \beta$ for growth and survival. Differences in average tumor volume of NT versus KRas or GSK3 $\alpha / \beta$-depleted MiaPaCa2 tumors were statistically significant starting at day 4 (Fig. $3 \mathrm{~d}$, e).
SB inhibits growth of mutant KRas xenografts from pancreatic cancer patients. To determine whether the GSK3 inhibitor can also inhibit growth of patient-derived xenografts (PDXs), we used fresh tumor biopsies from three pancreatic cancer patients. Patient 1 (G12C mutant KRas; poorly differentiated T3N1 stage IIB) was refractory to neoadjuvant FOLFIRINOX (oxaliplatin (Eloxatin ${ }^{\oplus}$ ), leucovirin, irinotecan, and fluorouracil)/radiation; patient 2 (G12V mutant KRas; poorly differentiated T3N1 stage IIB) had no therapy; and patient 3 (G12D mutant KRas; stage IV hepatic metastasis) progressed after gemcitabine (Gemzar)/ abraxane (albumin-bound or nab-paclitaxel)/xeloda (Capecitabine) with radiation. Freshly resected tumors were subcutaneously implanted in NSG mice as previously described ${ }^{15}$ and randomized into vehicle $(n=10)$ or SB $(n=9-10)$ groups for each of the PDXs. Throughout treatment, SB significantly inhibited tumor growth of all three PDXs (Fig. 3f-h). By the last day of vehicle treatment, PDXs from patients 1,2 , and 3 showed average growth of $531 \%, 492 \%$, and $522 \%$, respectively. In contrast, SB treatment resulted in only $179 \%, 232 \%$, and $207 \%$ growth, respectively (Fig. $3 \mathrm{f}-\mathrm{h})$. Differences in tumor growth between vehicle- and SB-treated mice were statistically significant starting at day 3 (patient 1) and day 2 (patients 2 and 3) (Fig. 3f-h and Supplementary Fig. $4 \mathrm{a}-\mathrm{c}$ ).

SB treatment of mice blocks c-Myc and $\beta$-catenin phosphorylation. To determine whether SB inhibited its target in vivo, we evaluated whether SB affected phosphorylation of the 
a
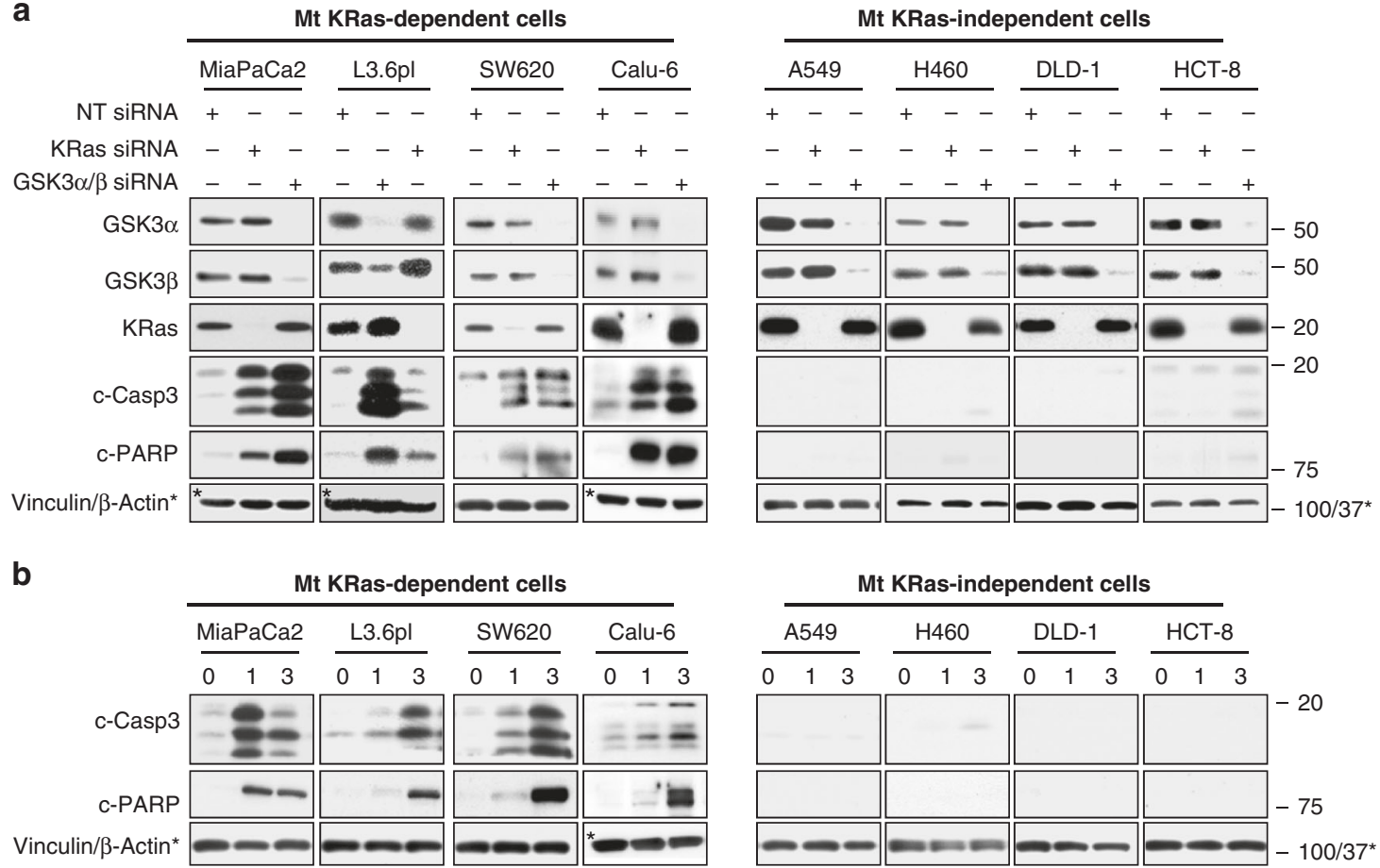

Fig. 2 Silencing of GSK3 $\alpha / \beta$ induces apoptosis only in mutant (Mt) KRas-dependent cancer cells. Mutant KRas-dependent (MiaPaCa2, L3.6pl, SW620, and Calu-6) and mutant KRas-independent (A549, H460, DLD-1, and HCT-8) human cancer cell lines were a transiently transfected with SMARTpool KRas, GSK3 $\alpha / \beta$, or NT siRNAs or $\mathbf{b}$ treated with the GSK3 $\alpha / \beta$ inhibitor SB and processed $72 \mathrm{~h}$ later for western blotting for KRas, GSK3 $\alpha$, GSK3 $\beta, \mathrm{c}-\mathrm{caspase}-3, \mathrm{c}-$ PARP, and Vinculin. For $\mathbf{a}$ and $\mathbf{b}$, experiments were repeated at least three times for each cell line

GSK3 substrates c-Myc and $\beta$-catenin in MiaPaCa2 and A549 xenografts. As shown in western blots, MiaPaCa2 but not A549 tumors from vehicle-treated mice contained high levels of phosphorylated c-Myc (Fig. 4a), as measured by an antibody that recognizes phospho-T58 c-Myc. T58 on c-Myc is phosphorylated by GSK3 ${ }^{16}$. Phosphorylation of c-Myc on T58 was blocked in $\mathrm{MiaPaCa} 2$ tumors from mice treated with $\mathrm{SB}$, indicating that $\mathrm{SB}$ reached its target in vivo. Similar to the c-Myc results, MiaPaCa2, but not A549, tumors from vehicle-treated mice contained high levels of phosphorylated $\beta$-catenin, as measured by an antibody that recognizes phospho-S33/S37/T41 $\beta$-catenin (Fig. 4a). $\beta$ Catenin phosphorylation by GSK3 on S33, S37, and T41 primes it for ubiquitination by the $\beta \operatorname{TrCP} \mathrm{E} 3$ ubiquitin ligase and destruction by the proteasome ${ }^{17}$. Accordingly, we observed total $\beta$-catenin levels to be extremely low in MiaPaCa2 but not in A549 tumors (Fig. 4a). Phosphorylation of $\beta$-catenin on S33, S37, and T41 was blocked in MiaPaCa2 tumors from mice treated with SB, resulting in increased accumulation of $\beta$-catenin. Importantly, and consistent with our cell culture results, SB treatment induced apoptosis in MiaPaCa2 but not in A549, tumors in vivo (Fig. 4a).

c-Myc and $\beta$-catenin contribute to antitumor activity of GSK3 blockade. The correlation between tumor growth inhibition, induction of apoptosis, and inhibition of c-Myc and $\beta$-catenin phosphorylation in MiaPaCa2 tumors in vivo suggested that cMyc and/or $\beta$-catenin may contribute to the antitumor activity of $\mathrm{SB}$. To test this hypothesis, we determined the effects of SB treatment $(1 \mu \mathrm{M})$ in MiaPaCa2 and A549 cells on phospho- $\beta$ catenin, phospho-c-Myc, and their corresponding total levels. Consistent with in vivo results (Fig. 4a), MiaPaCa2 cells had high levels of phospho-S33/S37/T41 $\beta$-catenin and little total $\beta$-catenin (Fig. 4b). In addition, $\beta$-catenin phosphorylation was rapidly (within $10 \mathrm{~min}$ ) blocked by $\mathrm{SB}$, and this blockade was paralleled by an accumulation of total $\beta$-catenin levels. A small and transient increase in phospho- $\beta$-catenin occurred between 8 and $24 \mathrm{~h}$, but the increase in total $\beta$-catenin was maintained. Similarly, SB rapidly decreased phospho-T58-c-Myc levels, which was paralleled by a modest increase in total c-Myc in the first $2 \mathrm{~h}$ followed by substantial increases at 4 and $8 \mathrm{~h}$. This is consistent with the observation that phosphorylation of T58 by GSK3 is associated with c-Myc degradation ${ }^{16}$. In contrast to the increase in $\beta$-catenin levels that was maintained until $72 \mathrm{~h}$, total c-Myc decreased after $8 \mathrm{~h}$ and was barely detectable by $72 \mathrm{~h}$ (Fig. $4 \mathrm{~b}$ ). This is consistent with in vivo results where long-term (21 days) SB treatment led to lower levels of c-Myc (Fig. 4a). In A549 cells, SB transiently decreased phospho- $\beta$-catenin at 10 and $30 \mathrm{~min}$ but this had little effect on the already high levels of total $\beta$-catenin (Fig. $4 \mathrm{~b}$ ). SB decreased phospho-c-Myc levels but had little effect on total levels, except a barely detectable effect after $24 \mathrm{~h}$. Consistent with the results shown in Figs. 1d, 2b, and 4a, SB induced apoptosis in MiaPaCa2 but not in A549 cells (Fig. 4b).

To further confirm whether the effects of SB on c-Myc and $\beta$ catenin levels are due to inhibition of GSK3, we have shown that depletion of GSK3 $\alpha / \beta$ with siRNA phenocopies the pharmacological inhibition of GSK3 $\alpha / \beta$ with SB by upregulating $\mathrm{c}-\mathrm{Myc}$ and $\beta$-catenin in MiaPaCa2 but not in A549 cells (Fig. 5a). We further reasoned that if SB inhibition of $\beta$-catenin and $\mathrm{c}-\mathrm{Myc}$ phosphorylation contributes to apoptosis induction, then forced expression of phosphorylation-deficient mutants of $\beta$-catenin and c-Myc should induce apoptosis in MiaPaCa2 but not in A549 cells. To this end, we infected these cells with GFP-lentivirus constructs containing mutant $\beta$-catenin that lacks the S33, S37, and T41 GSK3 phosphorylation sites and is therefore constitutively active ${ }^{18}$ or a T58A c-Myc mutant that cannot be phosphorylated by GSK3. After $96 \mathrm{~h}$, western blots showed 


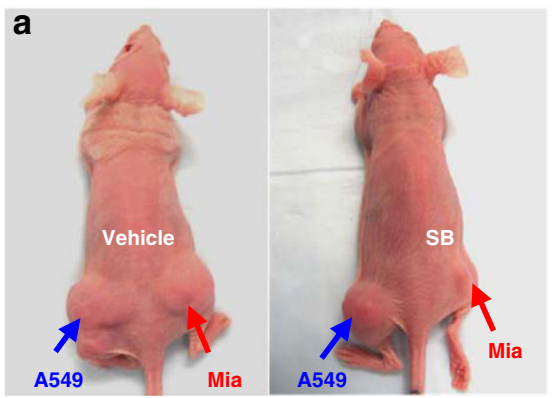

d

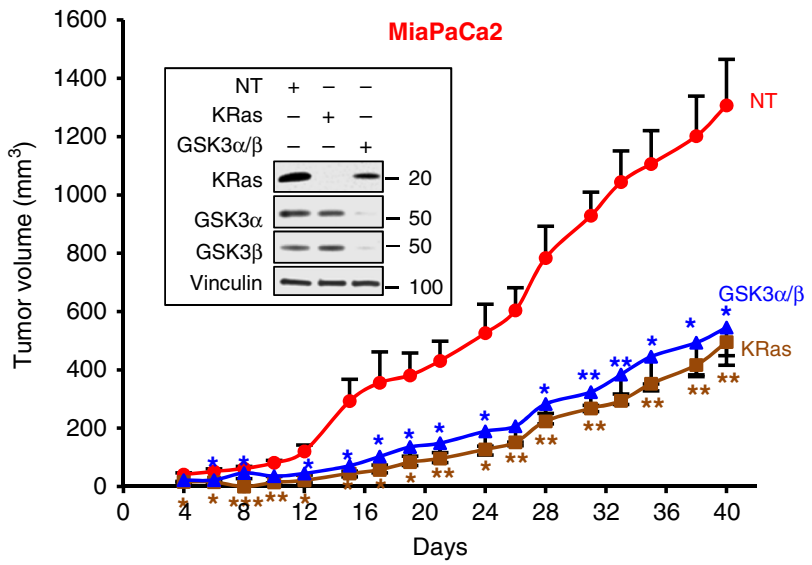

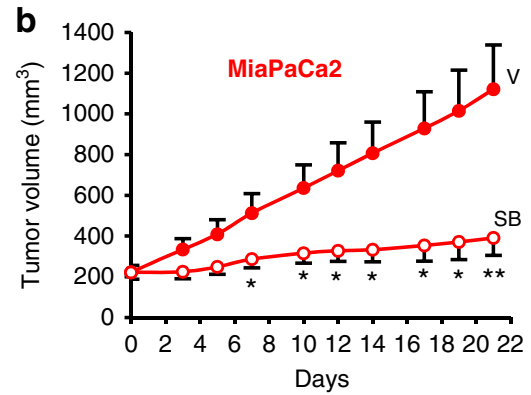

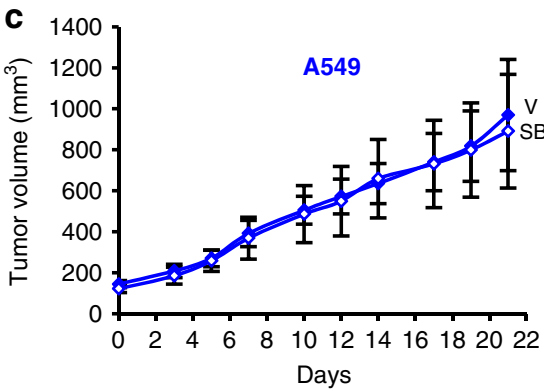

e
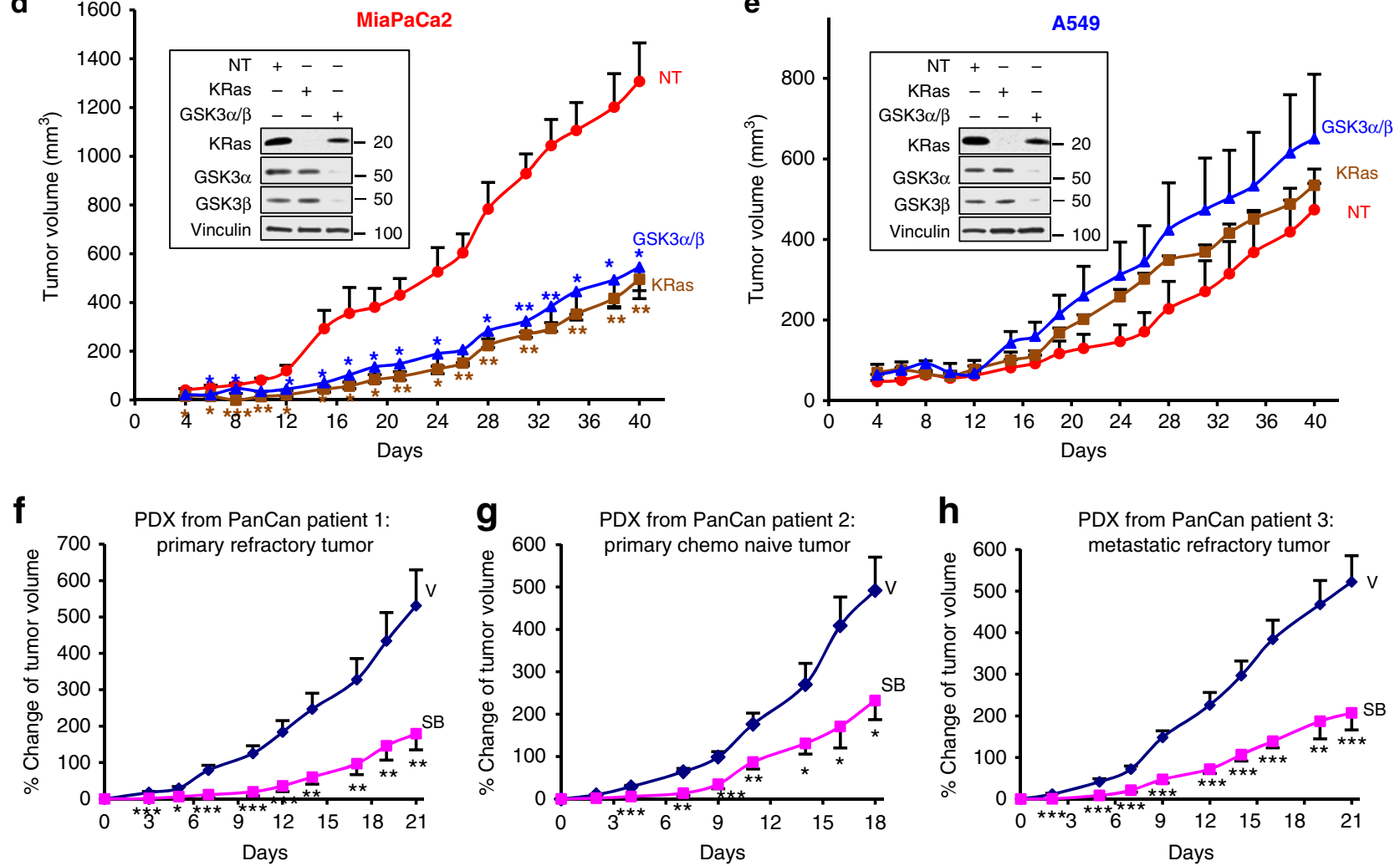

Fig. 3 SB suppresses mutant KRas-dependent tumors and pancreatic cancer patient xenografts. MiaPaCa2 and A549 cells were implanted on the right and left flank, respectively, and mice were treated daily with vehicle $(\mathrm{V})$ or SB $(50 \mathrm{mg} / \mathrm{kg})$. a Representative mice treated with either $\mathrm{V}$ or SB. b, c Effects of SB on the average tumor growth of all mice ( $\mathrm{V} ; 5$ mice and $\mathrm{SB} ; 6$ mice). Experiment was repeated three times. $\mathbf{d}$, e Average tumor volume of MiaPaCa2 and A549 tumors transfected with NT siRNA, KRas siRNA, or GSK3 $\alpha / \beta$ siRNA (NT; 3 mice, KRas sRNA; 3 mice, and GSK3 siRNA; 3 mice). Experiment done once. For $\mathbf{b}$ and $\mathbf{c}$, day 0 corresponds to day 14 after tumor implantation when the average tumor volumes were $150-200 \mathrm{~mm}^{3}$; for $\mathbf{d}$ and $\mathbf{e}$, day 0 corresponds to the day when the tumors were implanted. Insets: western blots confirming depletion of KRas and GSK3. $\mathbf{f}-\mathbf{h}$ Effects of vehicle and SB on the growth of patient-derived xenografts (PDXs) of chemotherapy/radiation-resistant primary (f), chemo-naive primary ( $(\mathbf{g})$, and chemo/radiation-resistant metastatic (h) tumors from pancreatic cancer patients. For $\mathbf{f}$ and $\mathbf{h}, \mathrm{V} ; 10$ mice and SB; 10 mice. For $\mathbf{g}, \mathrm{V} ; 10$ mice and SB; 9 mice) ( ${ }^{\star} P<0.05$; ${ }^{\star \star} P<0.01$; $\left.{ }^{\star \star \star} P<0.001\right)$. $P$ values determined by Student's $t$-test

that ectopic expression of mutant $\beta$-catenin and mutant cMyc induced apoptosis in MiaPaCa2 but not in A549 cells (Fig. 5b).

To investigate whether SB-induced apoptosis is mediated by cMyc and/or $\beta$-catenin, we determined whether CRISPR/Cas9targeted knockout of c-Myc or $\beta$-catenin rescues MiaPaCa2 cells from SB-induced apoptosis. After MiaPaCa2 cells were infected with lentivirus containing Cas9 and guide RNA (gRNA) to either $\beta$-catenin or c-Myc or scrambled for $48 \mathrm{~h}$ and subjected to antibiotic selection, we treated the resulting stable cells with $\mathrm{SB}$ for $48 \mathrm{~h}$. In scrambled gRNA-infected cells, SB inhibited c-Myc and $\beta$-catenin phosphorylation, upregulated $\mathrm{c}-\mathrm{Myc}$ and $\beta$-catenin, and induced caspase-3 activation and PARP cleavage (Fig. 6a). In contrast, targeted knockout of c-Myc and $\beta$-catenin in cells infected with c-Myc or $\beta$-catenin gRNA abrogated SB-induced apoptosis (Fig. 6a). Similar results were obtained with the other mutant KRas-dependent cells (Calu-6, L3.6pl, and SW620) where knocking out $\mathrm{c}-\mathrm{Myc}$ or $\beta$-catenin prevented SB from inducing apoptosis (Fig. 6b-d). Unlike mutant KRas-dependent cells, in A549 cells, SB did not induce apoptosis in scrambled gRNAinfected cells or in c-Myc- and $\beta$-catenin-knocked out cells (Fig. 6). Taken together, we found that pharmacological inhibition and gene silencing of GSK $3 \alpha / \beta$ selectively induced apoptosis and inhibited tumor growth of mutant KRas-dependent tumors, which was at least in part mediated by c-Myc and $\beta$ catenin. 
a

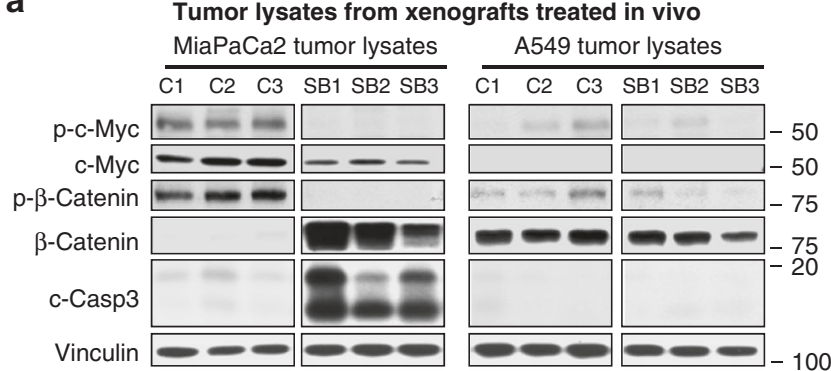

b
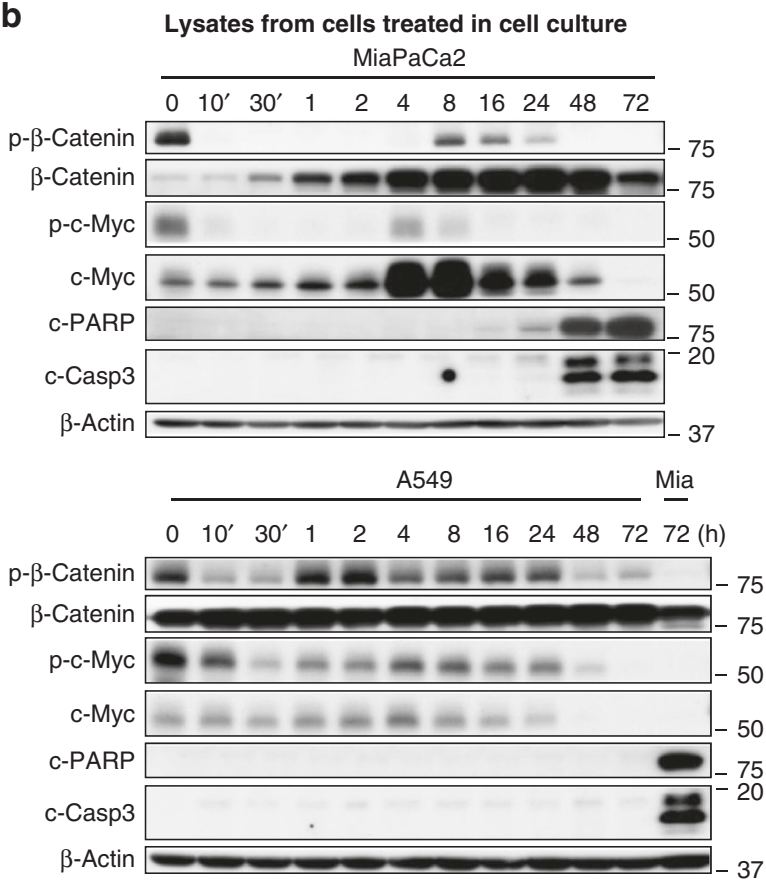

Fig. 4 SB blocks the phosphorylation of $\beta$-catenin S33/S37/T41 and T58 c-Myc. a Western blots of MiaPaCa2 and A549 tumor lysates from xenografts resected from mice treated with vehicle or $\mathrm{SB}$. C1, C2, and C3 are tumors from mice treated with vehicle control. SB1, SB2, and SB3 are tumors from mice treated with $S B$. Experiment was done once. $\mathbf{b}$ Western blot of MiaPaCa2 and A549 cells treated with $1 \mu \mathrm{M}$ SB for various lengths of time. Mia designates MiaPaCa2 lysates used as positive control. Experiment was done twice

\section{Discussion}

In this manuscript, we revealed that human tumors dependent on mutant KRas require GSK $3 \alpha / \beta$ for viability, survival, and tumor growth. In contrast, GSK $3 \alpha / \beta$ is dispensable for mutant KRasindependent tumors. Importantly, the in vivo growth of three PDXs from primary chemo-naive, primary refractory, and metastatic refractory pancreatic cancer patients was inhibited by the GSK $3 \alpha / \beta$ inhibitor SB. These results suggest that SB can overcome resistance to chemo- and radiation therapy since two of the fresh biopsies were resected from patients who progressed on FOLFIRINOX, gemcitabine, abraxane, xeloda, and/or radiation. The antitumor effects of SB were independent of the mutant KRas isoforms, which included G12D, G12V, and G12C (fresh biopsies) and G12D, G12V, G12C, and Q61K (cell lines). This is important as it has been suggested that the position and type of mutations in KRas influence the transforming capacity of mutant KRas proteins and drug responses of cancer patients. It is important to note that GSK3 inhibitors did not score in previous chemical screens. One possible reason for this is that unlike our chemical screen, the previous chemical screens were not designed specifically to identify compounds that inhibit mutant KRasharboring/KRas-dependent, but not mt KRas-harboring/KRasindependent human cancer cell lines. The second possible reason is that in all our genetic depletion and pharmacological inhibition studies, we have targeted simultaneously both GSK3 $\alpha$ and GSK3 $\beta$. Indeed, mutant KRas-dependent human cancer cells undergo apoptosis only when both GSK3 $\alpha$ and GSK3 $\beta$ are knocked down simultaneously (Supplementary Fig. 5). In contrast, knocking down GSK3 $\alpha$ or GSK3 $\beta$ individually did not induce apoptosis (Supplementary Fig. 5).

An important finding of our studies is that suppression of GSK3 $\alpha / \beta$ inhibits mutant KRas-dependent tumor growth at least in part by a c-Myc- and $\beta$-catenin-dependent mechanism. Consistent with this, depletion of GSK3 $\alpha / \beta$ increased c-Myc- and $\beta$ catenin levels; and forced expression of $\beta$-catenin or c-Myc mutants that lack GSK3 phosphorylation sites is sufficient to induce apoptosis. Strikingly, these findings suggest that in mutant KRas-dependent human tumors, GSK3 has pro-survival activity, whereas $\beta$-catenin and $c-M y c$ have pro-apoptotic activity. Although the roles for GSK $3 \alpha / \beta$ in promoting apoptosis are well documented, there is also evidence for its pro-survival function. The reasons for this bi-functional role of GSK3 in survival and apoptosis have been thus far poorly understood and mainly explained by context-specific effects regarding cell lineage, signaling circuits, and transformation status ${ }^{19}$. For example, GSK3 $\alpha /$ $\beta$ induces apoptosis during DNA damage, hypoxia, and endoplasmic reticulum stress ${ }^{19}$. However, in some cancers GSK3 is overexpressed $^{20}$, and promotes survival by several mechanisms, including activation of the canonical ${ }^{21}$ and non-canonical ${ }^{22}$ nuclear factor-kB pathways, inhibition of apoptotic signaling and caspase activation $^{23}$, and inhibition of c-Myc-induced apopto$\operatorname{sis}^{24}$. Our findings are consistent with a pro-survival role for GSK3 in mutant KRas-dependent tumors, where GSK3 inhibition leads to c-Myc- and $\beta$-catenin-dependent tumor suppression.

Although $\mathrm{c}-\mathrm{Myc}$ overexpression is associated with tumorigenesis, c-Myc can also induce apoptosis ${ }^{24,25}$. For example, c-Myc can induce apoptosis by stabilizing p53 through Foxo/ARF blockade of Mdm2 $2^{26}$ and by activating ATM kinase ${ }^{27}$, TRAIL ${ }^{28}$, or the pro-apoptotic protein $\mathrm{Bax}^{29}$. When we inhibited GSK3 with SB treatment, c-Myc levels were substantially increased within $4 \mathrm{~h}$ of treatment, and this coupled with the gene knockout results of Fig. 6, suggest that $\mathrm{c}-\mathrm{Myc}$ was necessary for SB to induce apoptosis in mutant KRas-dependent human cancer cells.

c-Myc is a known transcriptional target of $\beta$-catenin; therefore, SB could induce apoptosis by inhibiting the phosphorylation of $\beta$ catenin, leading to its stabilization and induction of c-Myc transcription. This is consistent with the data in Fig. $4 \mathrm{~b}$, where the increase in $\beta$-catenin is accompanied by modestly increased $c$ Myc levels at early time points. Alternatively, SB could directly induce $\mathrm{c}$-Myc in a $\beta$-catenin-independent manner by inhibiting its GSK3-dependent phosphorylation on T58. The significant increase in total c-Myc levels at 4 and $8 \mathrm{~h}$, beyond the initial modest increase at earlier time points (Fig. 4b), coupled with the fact that T58 phosphorylation promotes c-Myc degradation ${ }^{16}$, support this direct mechanism. Further support for this is provided by the demonstration that the T58A c-Myc mutant is able to induce apoptosis on its own.

Together, these findings suggest that in mutant KRasdependent human cancer cells, inactivating GSK $3 \alpha / \beta$ induces apoptosis in a $\beta$-catenin- and c-Myc-dependent manner. Our findings also suggest that during progression of some tumors, mutant KRas may lead to a GSK3 $\alpha / \beta$-dependent downregulation of $\beta$-catenin and/or c-Myc and that inhibition of GSK3 in these cells unleashes the pro-apoptotic action of $\beta$-catenin and/or cMyc. In contrast, GSK $3 \alpha / \beta$ inhibition did not upregulate $\beta$ - 
a

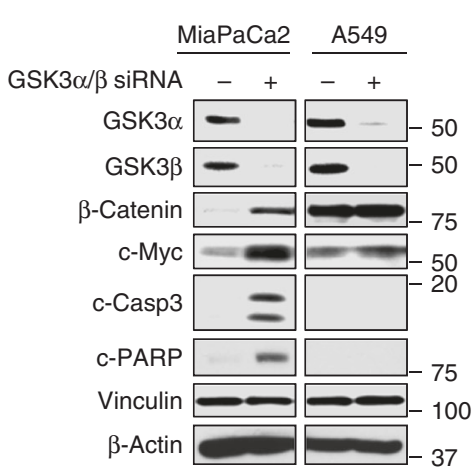

b

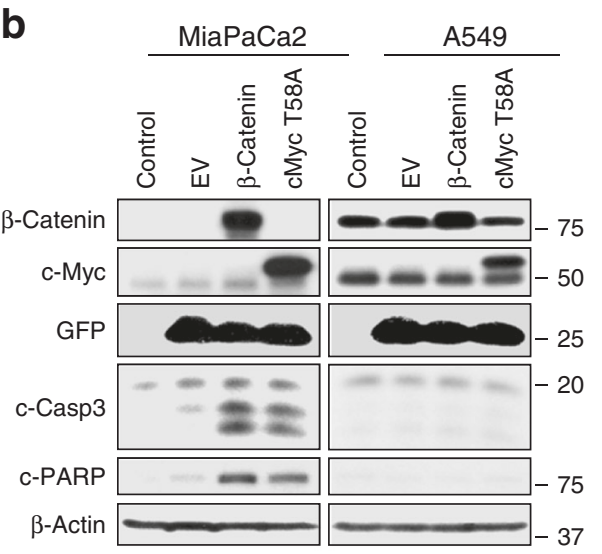

Fig. 5 a GSK $3 \alpha / \beta$ depletion induces $\beta$-catenin and c-Myc in MiaPaCa2 but not in A549 cells. The cells were transfected with GSK3- $\alpha / \beta$ siRNA or NT siRNA for $72 \mathrm{~h}$ and processed for western blotting with the indicated antibodies. Experiment was done three times. $\mathbf{b}$ Ectopic expression of CA $\beta$-catenin or T58A c-Myc induces apoptosis in MiaPaCa2 but not in A549 cells. Cells were infected with lentiviruses that contain mutant $\beta$-catenin that lacks the GSK3 phosphorylation sites, S33/S37/T41, and is therefore constitutively active, or mutant T58A c-Myc that cannot be phosphorylated by GSK3; cells were processed for western blotting after $96 \mathrm{~h}$. Experiment was done three times

a
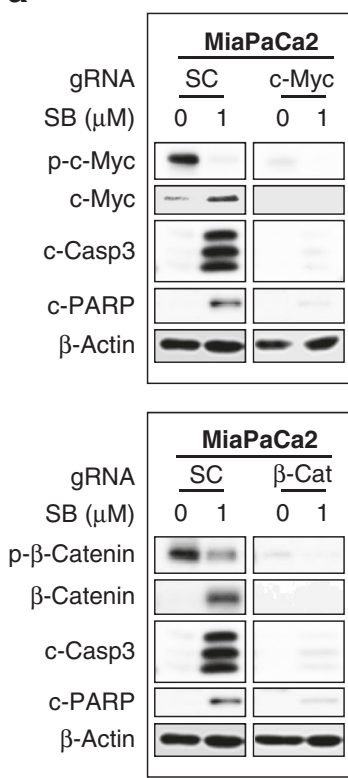

b

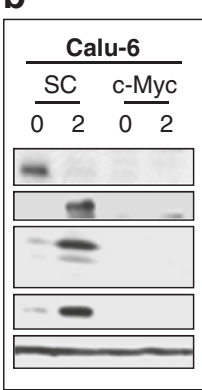

C
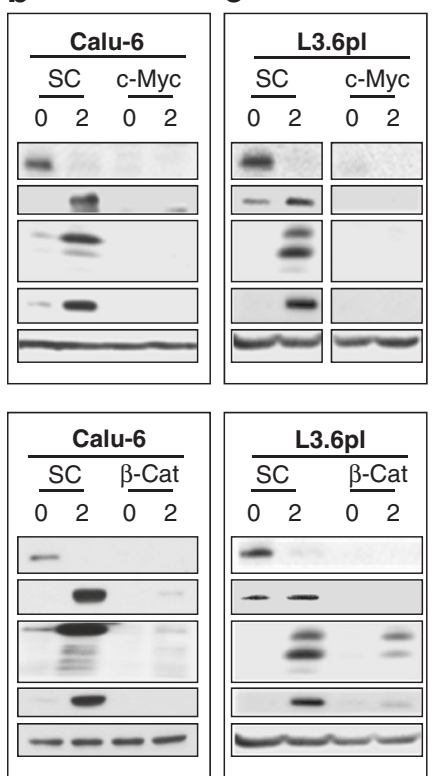

d
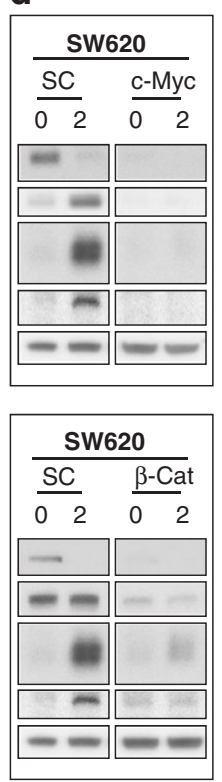

e

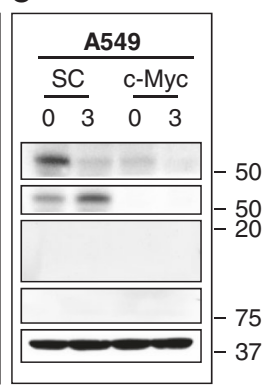

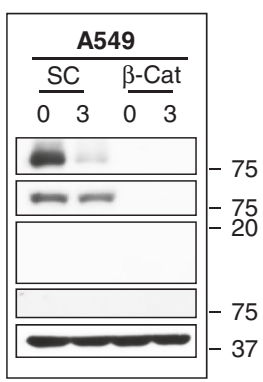

Fig. 6 CRISPR/Cas9 knockout of $\beta$-catenin or C-Myc rescues from SB-induced apoptosis. a-e Western blots of MiaPaCa2 (a), Calu-6 (b), L3.6pl (c), SW620 (d), and A549 (e) cells treated with SB at indicated concentrations for $48 \mathrm{~h}$ after knocking out c-Myc (top panel) or $\beta$-catenin (bottom panel) with lentiviral guide RNAs (gRNAs), including scrambled (SC), $\beta$-catenin ( $\beta$-Cat), and c-Myc gRNAs. Experiments were repeated at least three times for all cell lines except for SW620 and A549 where they were done once

catenin and c-Myc and did not induce apoptosis in mutant KRasindependent human tumors.

In summary, the novel mechanism presented here opens new avenues to therapeutically target mutant KRas-dependent human cancers by suppressing GSK3 $\alpha / \beta$, leading to $\beta$-catenin- and/or cMyc-dependent tumor abrogation. This significant finding has the potential to overcome the challenges that mutant KRas poses as a prevalent driver of human oncogenesis and therapy resistance. The GSK3 $\alpha / \beta$ inhibitor sensitivity of xenografts from primary and metastatic pancreatic cancer patients who progressed on chemo- and radiation therapies warrants further clinical investigation of GSK3 $\alpha / \beta$ inhibitors as single agents or in combination.

\section{Methods}

Cells lines, cell culture, and reagents. Human lung (Calu-6, A549, H460, H661, H2126, H322, H1299, H522, PC9, and H4006), colon (SW620, DLD-1, and HCT8), pancreatic (MiaPaCa2 and L3.6pl), and prostate (DU145) cancer cell lines and HEK293 cells were obtained from the American Type Culture Collection and cultured in Dulbecco's modified Eagle's medium (DMEM) or RPMI-1640 medium. Normal/immortalized T80 cells (J. Liu and R. Bast, MD Anderson Cancer Center) were cultured in Medium 199/MCDB 105. hTERT-immortalized HPNE cells (Channing Der, University of North Carolina) were grown in medium D (mixture of M3 medium and DMEM) containing one volume of M3 Base F culture medium (InCell Corp.), three volumes of glucose-free DMEM, $5.5 \mathrm{mM}$ glucose, $10 \mathrm{ng} / \mathrm{mL}$ $\mathrm{EGF}$, and $50 \mu \mathrm{g} / \mathrm{mL}$ gentamycin. All media were supplemented with $10 \%$ heatinactivated fetal bovine serum, $10 \mathrm{U} / \mathrm{mL}$ penicillin, and $10 \mu \mathrm{g} / \mathrm{mL}$ streptomycin. SB was synthesized in-house as described previously ${ }^{30}$ and dissolved in dimethyl sulfoxide (DMSO; Sigma-Aldrich). Tideglusib, AZD1080, and BIO were purchased from SelleckChem. The Published Kinase Inhibitor Set 1 (PKIS1) of 304 
compounds was received from GlaxoSmithKline (GSK). All cell lines were mycoplasma-free, monitored regularly with HEK-blue2 cells and mycoplasma detection kit from invivogen (cat\# rep-pt1).

Screening of PKIS1 library. KRas-dependent (MiaPaCa2) and -independent (A549) human cancer cell lines were screened with the 304 kinase inhibitor compounds of PKIS1 library to identify a kinase inhibitor that can selectively inhibit the viability of MiaPaCa2 over A549 cells using MTT assays. Cells were cultured in 96-well plates at a density of $3 \times 10^{3}$ cells/well and allowed to adhere overnight. The medium was then replaced with medium containing vehicle $(0.2 \%$ DMSO) or $1 \mu \mathrm{M}$ of each of 304 compounds for $72 \mathrm{~h}$ in one compound-one well format. After cells were incubated with $1 \mathrm{mg} / \mathrm{mL}$ MTT (Sigma), cell viability was quantified as described previously ${ }^{31}$.

Western blot analysis. To prepare whole-cell lysates, cells were washed twice with phosphate-buffered saline (PBS) and lysed on plates in Mammalian Protein Extraction Reagent (Thermos Scientific, catalog no. 78501) supplemented with protease inhibitor cocktail, $2 \mathrm{mM}$ phenylmethylsulfonyl fluoride, $2 \mathrm{mM} \mathrm{Na}_{3} \mathrm{VO}_{4}$, and $6.4 \mathrm{mg} / \mathrm{mL} p$-nitrophenylphosphate. Proteins from the lysates were separated by SDS-polyacrylamide gel electrophoresis and western blotted with the following antibodies: (a) from Santa Cruz: $\beta$-catenin (H-102; catalog no. sc-7199) and c-Myc (9E10, sc-40); (b) from Abcam: phospho-c-Myc (phospho-T58) (ab185655); (c) from Cell Signaling: GSK3a (D80E6; catalog no. 4337), GSK3ß (27c10; catalog no. 9315), cleaved caspase-3 (catalog no. 9664L), cleaved PARP (catalog no. 5625S), phospho- $\beta$-catenin (Ser 33/37/Thr41; catalog no. 9561); (d) from Calbiochem: antic-KRas (Ab-1; catalog no. OP24); and (e) from Sigma-Aldrich: vinculin (catalog no, V9131-.2ML) and anti- $\beta$-actin (catalog no. A5441).

Vector constructions and lentivirus production. Mutant T58A c-Myc was generated by mutagenesis PCR using forward primer T58A-F (TGCTGCCCGCCCCGCCCCTGTCCCCTAGCCGCC) and reverse primer T58AR (GGGGCGGGGCGGGCAGCAGCTCGAATTTC) (see Table 1 in the Supplementary Information) and wild-type c-Myc from MiaPaCa2 cells as template. For overexpression studies, T58A c-Myc was inserted in lentiviral pLEX-3FLAG-T2AGFP vector (modified from pLEX-MCS vector from Openbiosystem) between NotI and BamHI sites. Mutant $\beta$-catenin (pcDNA3 deltaN47 $\beta$-catenin, Addgene plasmid \#19287) was amplified by PCR and also constructed in pLEX-3FLAG-T2AGFP vector between NotI and BamHI sites.

To knockout c-Myc and $\beta$-catenin in human cancer cell lines, we constructed gRNAs in LentiCRISPRv2 vector (addgene \#52961 and \#83480) as described previously ${ }^{32}$ and tested their knockout efficiency in MiaPaCa2 cells by western blot. The most efficient gRNA vector was selected for knocking out c-Myc (gRNA sequence: CTGCTCGCCCTCCTACGTTG) and $\beta$-catenin (gRNA sequence: TCCCACTAATGTCCAGCGTT) in all cell lines (see Table 1 in the Supplementary Information). The scramble gRNA (GCACTACCAGAGCTAACTCA) was also inserted into LentiCRISPRv2 vector and served as negative control for all cell lines.

For lentivirus production, the lentiviral vector $(10 \mu \mathrm{g})$ and the two packaging viral vectors, pMD. $2 \mathrm{G}(5 \mu \mathrm{g})$ and pspPax2 $(5 \mu \mathrm{g})$, were co-transfected into 293T cells using polyethylenimine $(25 \mathrm{kDa}, 1 \mu \mathrm{g} / \mu \mathrm{L})^{33,34}$. The virus supernatant was collected at $48 \mathrm{~h}$ and concentrated at 1:100 ratio using Lenti- $\mathrm{X}^{\mathrm{sm}}$ Concentrator (TaKaRa/Clontech, \#631231). Viral RNA genome copies/mL were determined using Lenti-X ${ }^{\mathrm{m}}$ qRT-PCR Titration Kit (TaKaRa/Clontech).

gRNA-mediated knockout of $\beta$-catenin and c-Myc and SB rescue experiments. To generate stable pools, the cells infected with lentivirus containing scrambled gRNA, c-Myc gRNA, or $\beta$-catenin gRNA were selected with antibiotics (puromycin or blasticidin) for 5 days before pooling together and SB treatments. To determine whether knocking out c-Myc or $\beta$-catenin rescues from SB-induced apoptosis, these stable pools were seeded into six-well plates at $0.3 \times 10^{6}$ cells/well and treated with SB for $48 \mathrm{~h}$ at $1 \mu \mathrm{M}$ for MiaPaCa2, $2 \mu \mathrm{M}$ for Calu-6, L3.6pl, and SW620, and 3 $\mu \mathrm{M}$ for A549 cells. It is important to use early passage (passage 1 or 2 after stable pool generation) for these experiments. Western blots were performed as described above to detect phospho- $\beta$-catenin (Ser 33/37/Thr41), $\beta$-catenin, phospho-c-Myc (T58), c-Myc, cleaved PARP, cleaved caspase-3, and $\beta$-actin.

siRNA-mediated knockdown of KRas and GSK3 $\boldsymbol{\alpha} / \boldsymbol{\beta}$. After cells were plated onto 6-well plates for western blotting and then 96 -well plates for cell viability assays for $24 \mathrm{~h}$, they were transiently transfected using the Lipofectamine RNAiMAX reagent (catalog no. 13778; Invitrogen, Carlsbad, CA) with 20 nM SMARTpool Human KRas siRNA (catalog no. M-005069-00; Dharmacon), SMARTpool NT siRNA \#2 (catalog no. D-001206-14-05; Dharmacon), SignalSilence GSK3 $\alpha / \beta$ siRNA (catalog no. 6301; Cell Signaling), and SignalSilence control siRNA (unconjugated; catalog no. 6568; Cell Signaling). Transfected cells were collected 48 or $72 \mathrm{~h}$ after transfection for western blot analysis as described above, and after $72 \mathrm{~h}$ for CellTiter-Glo ${ }^{\circ}$ Luminescent Cell Viability Assay (Promega, Madison, WI, USA) as described below.
Cell viability assay. Cell viability assays were carried out using the CellTiter-Glo Luminescent Cell Viability Assay (Promega, Madison, WI). Cells were seeded in 96-well plates at a density of $3 \times 10^{3}$ to $4 \times 10^{3}$ cells/well, allowed to adhere overnight, and treated with vehicle (DMSO) or drug for $72 \mathrm{~h}$, after which they were processed for viability using CellTiter-Glo reagent. Each condition was performed in replicates of 6 wells.

Antitumor studies of human tumor xenografts in nude mice. Female athymic nude mice were purchased from Charles River Laboratories (Wilmington, MA). The mice were housed, maintained, and treated in accordance with the Institutional Animal Care and Use Committee procedures and guidelines. Exponentially growing A549 and MiaPaCa2 cells were harvested via trypsinization, pelleted at $300 \times g$ for $5 \mathrm{~min}$, resuspended in sterile Dulbecco's PBS (DPBS; Invitrogen) at $5 \times$ $10^{6}$ cells $(\mathrm{MiaPaCa} 2)$ and $10 \times 10^{6}$ (A549) cells per $100 \mu \mathrm{L}$, and injected into right and left flank, respectively, of each mouse. The tumor xenografts were monitored with an electronic caliper three times per week. Tumor volume was calculated using the following formula: volume $=\left(L^{2} W\right) / 2$, where $L$ is length and $W$ is width, with width defined as the largest measurement and length is the smallest measurement. When the tumors reached $150-200 \mathrm{~mm}^{3}$, animals were randomized and treatment schedules (control or SB) were implemented. The vehicle received $10 \%$ DMSO, 50\% PEG (MW 300 from MP Biochemicals, LLC, Solon, OH), and 40\% $\mathrm{dH} 2 \mathrm{O}$; the SB group received $50 \mathrm{mg} / \mathrm{kg} /$ day of SB-maleic acid salt. Vehicle and SB $(100 \mu \mathrm{L})$ were administered by intraperitoneal injections once daily.

To determine the effects of depleting KRas and GSK $3 \alpha / \beta$ on the growth of MiaPaCa2 and A549 cells, these cells were transiently transfected with $20 \mathrm{nM}$ of SMARTpool siGENOME human KRas, GSK3 $\alpha / \beta$, and NT siRNA as described above for $48 \mathrm{~h}$, then harvested, washed twice in ice-cold sterile DPBS (Invitrogen), counted for viable cells by trypan blue exclusion, resuspended in sterile DPBS at $5 \times 10^{6}$ cells (MiaPaCa2) and $10 \times 10^{6}$ (A549) cells/100 $\mu \mathrm{L}$, and injected into right and left flanks, respectively, of each mouse. A portion of these transiently transfected cells were collected to run for western blot analysis to confirm the knockdown of target genes by siRNA. The tumor xenografts were monitored, and the volumes were determined three times a week as described above.

\section{Antitumor efficacy studies of PDXs of tumors from pancreatic cancer}

patients. To determine whether pharmacological inhibition of GSK3 can inhibit the growth of PDXs, we obtained fresh tumor biopsies from three pancreatic cancer patients (University of Florida, IRB protocol \# 201600873). Patient 1 (G64) (G12C KRas mutation), who showed poorly differentiated T3N1 pancreatic cancer (stage IIB), completed 6 weeks of neoadjuvant FOLFIRINOX with 1 week of radiation. Therapy was concluded 5 weeks before surgery. CA 19-9 biomarker levels declined from 500 to 74 during this time, but final pathology demonstrated no tumor necrosis (poor histopathologic response). Patient 2 (G80) (G12V KRas mutation), who also showed poorly differentiated T3N1 pancreatic cancer (stage IIB), received no neoadjuvant therapy. Patient 3 (LM3) (G12D KRas mutation), who had hepatic metastatic pancreatic cancer (stage IV), progressed after neoadjuvant therapy with gemcitabine, abraxane, and xeloda with radiation. Therapy duration was 6 weeks and was completed 6 weeks before surgery.

Upon pancreatic tumor resection, fresh 2-mm tumor pieces were obtained and transported on ice to the animal surgery suite for subcutaneous implantation into NOD.Cg-Prkdcscid Il2rgtm1Wjl/SzJ (NSG) mice. The mice were housed, maintained, and treated in accordance with the Institutional Animal Care and Use Committee procedures and guidelines (protocol \#201406590). Incisions of $1 \mathrm{~cm}$ were made on right flanks of anesthetized NSG mice, and blunt dissection of the subcutaneous layer was performed. A viable tumor piece was placed in the flank subcutaneous tissue, and the skin was closed with surgical clips (generation 1). Once engrafted and tumors reached end point $(1.5 \mathrm{~cm}$ in diameter), tumors were divided evenly into 2 - $\mathrm{mm}$ pieces and re-implanted into NSG mice as above (generation 2). Generation 3 was generated similarly (details as described in ref. ${ }^{15}$ ). When the tumors from early-passage (generation 2 or 3 ) mice reached approximately $100-200 \mathrm{~mm}^{3}$, the animals were randomized into vehicle and SB treatment groups as described above for the cell line xenograft models.

Ectopic expression of $\boldsymbol{\beta}$-catenin and c-Myc. The day before infection, $2 \times 10^{5}$ cells were seeded per well of six-well plates. Cells were plated overnight to reach $70-80 \%$ confluence and were then infected with concentrated lentiviral $\left(200 \times 10^{8}\right.$ copies total) GFP-EV, GFP mutant $\beta$-catenin ${ }^{35}$, and mutant GFP-T58A c-Myc ${ }^{25,36}$ Infected cells were harvested after $96 \mathrm{~h}$ and processed for western blot analysis.

Statistical tests. All $P$ values in this manuscript were determined by Student's $t$ test. All error bars in figures represent standard errors.

\section{Data availability}

All data generated or analyzed during this study are included in this published article (and its Supplementary Information files). 
Received: 9 January 2018 Accepted: 15 November 2018

Published online: 04 December 2018

\section{References}

1. Hanahan, D. \& Weinberg, R. A. Hallmarks of cancer: the next generation. Cell 144, 646-674 (2011).

2. Pylayeva-Gupta, Y., Grabocka, E. \& Bar-Sagi, D. RAS oncogenes: weaving a tumorigenic web. Nat. Rev. Cancer 11, 761-774 (2011).

3. Slebos, R. J. et al. K-ras oncogene activation as a prognostic marker in adenocarcinoma of the lung. N. Engl. J. Med. 323, 561-565 (1990),

4. Chetty, R. \& Govender, D. Gene of the month: KRAS. J. Clin. Pathol. 66, 548-550 (2013).

5. Pao, W. et al. KRAS mutations and primary resistance of lung adenocarcinomas to gefitinib or erlotinib. PLoS Med. 2, e17 (2005).

6. Lievre, A. et al. KRAS mutation status is predictive of response to cetuximab therapy in colorectal cancer. Cancer Res. 66, 3992-3995 (2006)

7. Ostrem, J. M. \& Shokat, K. M. Direct small-molecule inhibitors of KRAS: from structural insights to mechanism-based design. Nat. Rev. Drug Discov. 15, 771-785 (2016).

8. Welsch, M. E. et al. Multivalent small-molecule pan-RAS inhibitors. Cell 168, 878 (2017).

9. McCormick, F. Targeting KRas directly. Annu. Rev. Cancer Biol. 2, 81-90 (2018).

10. Cox, A. D., Fesik, S. W., Kimmelman, A. C., Luo, J. \& Der, C. J. Drugging the undruggable RAS: mission possible? Nat. Rev. Drug Discov. 13, 828-851 (2014).

11. Downward, J. RAS synthetic lethal screens revisited: still seeking the elusive prize? Clin. Cancer Res. 21, 1802-1809 (2015).

12. Elkins, J. M. et al. Comprehensive characterization of the published kinase inhibitor set. Nat. Biotechnol. 34, 95-103 (2016).

13. Fleming, J. B., Shen, G. L., Holloway, S. E., Davis, M. \& Brekken, R. A. Molecular consequences of silencing mutant K-ras in pancreatic cancer cells: justification for K-ras-directed therapy. Mol. Cancer Res. 3, 413-423 (2005).

14. Singh, A. et al. A gene expression signature associated with "K-Ras addiction" reveals regulators of EMT and tumor cell survival. Cancer Cell 15, 489-500 (2009).

15. Delitto, D. et al. Patient-derived xenograft models for pancreatic adenocarcinoma demonstrate retention of tumor morphology through incorporation of murine stromal elements. Am. J. Pathol. 185, 1297-1303 (2015).

16. Sears, R. et al. Multiple Ras-dependent phosphorylation pathways regulate Myc protein stability. Genes Dev. 14, 2501-2514 (2000).

17. Liu, C. et al. Control of b-catenin phosphorylation/degradation by a dualkinase mechanism. Cell 108, 837-847 (2002).

18. Tetsu, O. \& McCormick, F. b-Catenin regulates expression of cyclin D1 in colon carcinoma cells. Nature 398, 422-426 (1999).

19. Jacobs, K. M. et al. GSK-3: a bifunctional role in cell death pathways. Int. J. Cell Biol. 2012, 1-11 (2012).

20. Shakoori, A. et al. Deregulated GSK3 $\beta$ activity in colorectal cancer: its association with tumor cell survival and proliferation. Biochem. Biophys. Res. Commun. 334, 1365-1373 (2005).

21. Demarchi, F., Bertoli, C., Sandy, P. \& Schneider, C. Glycogen synthase kinase$3 \beta$ regulates NF-kB1/p105 stability. J. Biol. Chem. 278, 39583-39590 (2003).

22. Bang, D., Wilson, W., Ryan, M., Yeh, J. J. \& Baldwin, A. S. GSK-3a promotes oncogenic KRAS function in pancreatic cancer via TAK1-TAB stabilization and regulation of non-canonical NF-кB. Cancer Discov. 3, 690-703 (2013).

23. Sun, M., Song, L., Li, Y., Zhou, T. \& Jope, R. S. Identification of an antiapoptotic protein complex at death receptors. Cell Death Differ. 15 , 1887-1900 (2008).

24. Kotliarova, S. et al. Glycogen synthase kinase 3 inhibition induces glioma cell death through c-MYC, NF-kB and glucose regulation. Cancer Res. 68 , 6643-6651 (2008).

25. Askew, D. S., Ashmun, R. A., Simmons, B. C. \& Cleveland, J. L. Constitutive cmyc expression in an IL-3-dependent myeloid cell line suppresses cell cycle arrest and accelerates apoptosis. Oncogene 6, 1915-1922 (1991).

26. Zindy, F. et al. Myc signaling via the ARF tumor suppressor regulates p53-dependent apoptosis and immortalization. Genes Dev. 12, 2424-2433 (1998).

27. Pusapati, R. V. et al. ATM promotes apoptosis and suppresses tumorigenesis in response to Myc. Proc. Natl Acad. Sci. USA 103, 1446-1451 (2006).

28. Nieminen, A. L., Partanen, J. I., Jau, A. \& Klefstrom, J. c-Myc primed mitochondria determine cellular sensitivity to TRAIL-induced apoptosis. EMBO J. 26, 1055-1067 (2007).
29. Mitchell, K. O. et al. Bax is a transcriptional target and mediator of c-Mycinduced apoptosis. Cancer Res. 60, 6318-6325 (2000).

30. Witherington, J. et al. 6-Aryl-pyrazolo[3,4-b]pyridines: potent inhibitors of glycogen synthase kinase-3 (GSK-3). Bioorg. Med. Chem. Lett. 13, 3055-3057 (2003).

31. Falsetti, S. C. et al. Geranylgeranyltransferase I inhibitors target RalB to inhibit anchorage-dependent growth and induce apoptosis and RalA to inhibit anchorage-independent growth. Mol. Cell. Biol. 27, 8003-8014 (2007).

32. Sanjana, N. E., Shalem, O. \& Zhang, F. Improved vectors and genome-wide libraries for CRISPR screening. Nat. Methods 11, 783-784 (2014).

33. Tang, Y., Garson, K., Li, L. \& Vander Hyden, B. C. Optimization of lentiviral vector production using polyethylenimine-mediated transfection. Oncol. Lett. 9, 55-62 (2015).

34. Kutner, R. H., Zhang, X. Y. \& Reiser, J. Production, concentration and titration of pseudotyped HIV-1-based lentiviral vectors. Nat. Protoc. 4, 495-505 (2009).

35. Kolligs, F. T., Hu, G., Dang, C. V. \& Fearon, E. R. Neoplastic transformation of RK3E by mutant $\beta$-catenin requires deregulation of Tcf/Lef transcription but not activation of c-myc expression. Mol. Cell. Biol. 19, 5696-5706 (1999).

36. Yang, H. et al. Aurora-A regulates telomerase activity through c-Myc in human ovarian and breast epithelial cells. Cancer Res. 64, 463-467 (2004).

\section{Acknowledgements}

This work was funded in part by NIH grant R35 CA197731-01 (Said Sebti, Ph.D.) and was supported in part by the Chemical Biology, Molecular Genomics, and SAIL Core Facilities at the H. Lee Moffitt Cancer Center \& Research Institute; an NCI-designated Comprehensive Cancer Center (P30-CA076292). We thank these cores for their outstanding assistance and expertise. We also thank David H. Drewry and colleagues, GlaxoSmithKline, and the UNC Eshelman School of Pharmacy, Division of Chemical Biology and Medicinal Chemistry, for providing us with the Published Kinase Inhibitor Set 1 (PKIS1). We thank Yunting Luo (Chemical Biology Core, Moffitt Cancer Center) for synthesis of some of the SB compound. The synthesis of some of the SB compound was in part funded by NIH grant R50CA211447 (Harshani R. Lawrence). We also thank Alan List, Tom Sellers, John Cleveland, Eric Haura, and Uwe Rix (Moffitt Cancer Center) for critical reading of this manuscript, and Rasa Hamilton for editorial assistance.

\section{Author contributions}

A.K., S.X., H.Y., and P.K. performed experiments, collected data, and prepared the figures. M.A. and H.R.L. synthesized, purified, and characterized the SB compound. J.T. resected fresh tumor biopsies from pancreatic cancer patients. D.D. generated patientderived xenografts in mice under the supervision of J.T. R.H.Y.J. analyzed data. S.M.S. designed experiments, supervised the work, and wrote the manuscript. All authors reviewed the manuscript.

\section{Additional information}

Supplementary Information accompanies this paper at https://doi.org/10.1038/s41467 018-07644-6.

Competing interests: The authors declare no competing interests.

Reprints and permission information is available online at http://npg.nature.com/ reprintsandpermissions/

Publisher's note: Springer Nature remains neutral with regard to jurisdictional claims in published maps and institutional affiliations.

\footnotetext{
Open Access This article is licensed under a Creative Commons Attribution 4.0 International License, which permits use, sharing, adaptation, distribution and reproduction in any medium or format, as long as you give appropriate credit to the original author(s) and the source, provide a link to the Creative Commons license, and indicate if changes were made. The images or other third party material in this article are included in the article's Creative Commons license, unless indicated otherwise in a credit line to the material. If material is not included in the article's Creative Commons license and your intended use is not permitted by statutory regulation or exceeds the permitted use, you will need to obtain permission directly from the copyright holder. To view a copy of this license, visit http://creativecommons.org/ licenses/by/4.0/.
}

(c) The Author(s) 2018 